\title{
Safety and effectiveness of a new enzyme-targeting radiosensitization treatment (KORTUC II) for intratumoral injection for low-LET radioresistant tumors
}

\author{
YASUHIRO OGAWA $^{1}$, KEI KUBOTA ${ }^{1}$, HIRONOBU UE ${ }^{1}$, MICHIKO TADOKORO $^{1}$, RINA MATSUI ${ }^{1}$, \\ TOMOAKI YAMANISHI $^{1}$, NORIHIKO HAMADA ${ }^{1}$, SHINJI KARIYA ${ }^{1}$, AKIHITO NISHIOKA $^{1}$, \\ HIDEKI NAKAJIMA $^{2}$, MASAHITO TARUTANI ${ }^{2}$ and SHIGETOSHI SANO ${ }^{2}$
}

\author{
${ }^{1}$ Department of Diagnostic Radiology and Radiation Oncology, Medical School, Kochi University, Nankoku, Kochi \\ 783-8505; ${ }^{2}$ Department of Dermatology, Medical School, Kochi University, Nankoku, Kochi 783-8505, Japan
}

Received January 13, 2011; Accepted March 10, 2011

DOI: 10.3892/ijo.2011.1069

\begin{abstract}
Linear accelerator-based radiotherapy has little effect on tumors such as malignant melanoma, various types of sarcoma, and most locally-advanced neoplasms that have grown to several centimeters or more. These tumors contain many hypoxic cancer cells or large amounts of anti-oxidative enzymes, and are therefore resistant to low linear energy transfer radiation. Therefore, it was necessary to develop a new radiosensitizer to overcome these situations. We previously developed a new enzyme-targeting radiosensitization treatment named KORTUC I, which uses 3\% w/v hydrogen peroxide solution-soaked gauze. We developed a new radiosensitizer for intratumoral injection (KORTUC II), comprising a combination of hydrogen peroxide and sodium hyaluronate. After providing a fully informed written consent, 52 patients with unresectable or recurrent neoplasms (53 lesions) were enrolled in the KORTUC II trial. The present study of 52 patients with unresectable or recurrent neoplasms showed that KORTUC II is safe when injected intratumorally, well tolerated, and can efficiently exert a radiation sensitizing effect. Because this radiosensitizer is safe and less expensive than other methods, and can be applied for almost every type of low-LET radioresistant neoplasm, it has potential for worldwide and immediate use.
\end{abstract}

\section{Introduction}

Radiotherapy is second to surgery as a local treatment method for malignant tumors. Because it can be applied to elderly

Correspondence to: Professor Yasuhiro Ogawa, Department of Diagnostic Radiology and Radiation Oncology, Medical School, Kochi University, Oko-cho, Nankoku-shi, Kochi Prefecture 783-8505, Japan E-mail: ogaway@kochi-u.ac.jp

Key words: KORTUC, radiosensitizer, hydrogen peroxide, radiosensitization, sodium hyaluronate, breast cancer, intratumoral injection, ultrasound patients and also enables normal organs and tissues to be conserved, its use has spread rapidly in recent years. However, linear accelerator (Linac) generated high-energy X-rays and electron beams that are generally used for radiotherapy are low linear energy transfer (LET) forms of radiation and therefore have a relatively weak biological effect. Accordingly, linear accelerator based radiotherapy has little effect on tumors such as malignant melanoma, various types of sarcoma, and glioblastoma multiforme. Further, because locally advanced neoplasms that have grown to several centimeters or more contain many hypoxic cancer cells or large amounts of anti-oxidative enzymes, and are therefore resistant to low-LET radiation, they are not amenable to linear accelerator based radiotherapy.

Heavy particle beam therapy is a method used to improve the radiotherapeutic effect in these situations. However, the widespread use of this therapy is hampered by the cost of the equipment, which involves an investment of several tens of billions of yen.

In the past 40 years, various radiosensitizers (such as metronidazole, misonidazole, etanidazole, and nimorazole) have been developed to increase the radiotherapeutic effect (1-3). However, these agents have not yet been approved for clinical practice because their effects are uncertain and they have side effects such as peripheral neuropathy.

When pre-treating cancer tissue for immunohistochemical staining, peroxidase blockade is usually an essential step to avoid non-specific staining of the tissue. Most cancer tissues contain abundant peroxidase/catalase, and these anti-oxidative enzymes are easily inactivated by low concentrations of hydrogen peroxide (approximately $0.3 \%$ ) resulting in microbubbles of oxygen being produced. Pre-treatment with hydrogen peroxide inactivates peroxidase/catalase, resulting in negative staining for peroxidase activity. This can produce ideal circumstances for clinical radiotherapy using low-LET radiation from linear accelerators. As tumors in this state are considered ideal for low-LET radiotherapy in terms of existence of abundant oxygen and inactivation of anti-oxidative enzymes, it is desirable to reproduce these circumstances when performing clinical radiotherapy using low-LET radiation from currently available linear accelerators. 
Using radioresistant osteosarcoma cell lines (HS-Os-1) and prostatic cancer cells, we previously confirmed that sensitivity to $\mathrm{X}$-ray radiation could be increased and apoptosis rapidly induced by adding a low concentration of hydrogen peroxide to the culture medium during irradiation (4-7). Normally, such radioresistant osteosarcoma cells and chondrocytes show little reactive oxygen species formation, even when exposed to radiation of $30 \mathrm{~Gy}(5,8)$. In contrast, when irradiated in the presence of a low concentration $(0.1 \mathrm{mM}$, for example) of hydrogen peroxide, reactive oxygen species are clearly produced in cells exposed to radiation in the order of $10 \mathrm{~Gy}$, giving rise to apoptosis (5). On the basis of the above-mentioned reasoning, we postulated that the osteosarcoma cells were X-ray radiation resistant because they contained peroxidase and catalase (6). We theorized that when irradiation is conducted in the presence of hydrogen peroxide, the action of the anti-oxidative enzymes is blocked and oxygen is produced, oxidizing the X-ray radioresistant hypoxic cells, thereby rendering them highly sensitive to radiation $(7,8)$.

We previously developed KORTUC I (Kochi OxydolRadiation Therapy for Unresectable Carcinomas, Type I), a radiosensitization method for the body surface which remarkably enhances the radiosensitivity of various types of superficially exposed and locally advanced neoplasms (9). In KORTUC I, we used sterilized cotton soaked in a $3 \% \mathrm{w} / \mathrm{v}$ hydrogen peroxide solution (Oxydol) as a bolus and then applied irradiation with a $15-\mathrm{MeV}$ electron beam from a linear accelerator. Radiotherapy was conducted three times a week, with each radiation dose amounting to $4 \mathrm{~Gy}$, for a total radiation dose of $48 \mathrm{~Gy}$. Good effects were achieved, with complete disappearance of the tumor (Fig. 1).

However, this hydrogen peroxide solution is a strong irritant that should not be applied directly in large quantities to an affected area. Its use is also contraindicated where the hydrogen peroxide solution could seep into a body cavity, such as in the case of fistula or contused wound. Moreover, hydrogen peroxide is unstable, and when applied to the affected area, it immediately starts releasing oxygen. Hence, if it were to be injected subcutaneously or intratumorally as is, there is a danger that it would be rapidly degraded to water and oxygen by the action of anti-oxidative enzymes such as peroxidase and catalase. This could cause complications such as pulmonary embolism. For this reason, there is a need for a pharmaceutical formulation that can alleviate the irritation of hydrogen peroxide so that it can be applied to fistulas or contused wounds or safely injected into the human body, and that also delays and suppresses the degradation of the hydrogen peroxide.

After much experimentation, we found that irritation of the skin, mucous membranes, and other tissues by hydrogen peroxide could be reduced by combining sodium hyaluronate with hydrogen peroxide (10). Moreover, this coadministration of sodium hyaluronate reduced the tendency of the hydrogen peroxide to readily give off oxygen due to its instability. Thus, even when injected into the human body, its rapid degradation due to the action of anti-oxidative enzymes was significantly reduced, enabling the radiosensitizer effect to be exerted more safely and efficiently and for a longer duration.

Based on our clinical experiences using KORTUC I and these experimental results, we therefore developed KORTUC II, a new radiosensitizer containing hydrogen peroxide and sodium hyaluronate for intratumoral injection for various types of tumors that are not superficially exposed (11). The concepts underlying this new enzyme-targeting radiosensitization treatment are shown in Fig. 2. The aim was to formulate an efficient low-LET radiosensitizer that has minimal irritation, can be safely injected into the human body, and can delay or reduce the degradation of the hydrogen peroxide and preserve the oxygen concentration in the tumor tissue for more than $24 \mathrm{~h}$.

The purpose of this study was to evaluate the safety and effectiveness of KORTUC II for patients with recurrent and/or unresectable neoplasms.

\section{Materials and methods}

Patients treated with KORTUC II. After providing fully informed written consent, 52 patients (53 lesions) were enrolled in the KORTUC II trial. Thirty-one had unresectable or recurrent breast cancer (including elderly patients), 8 had unresectable or recurrent soft tissue neoplasms, 4 had cervical lymph node metastases, and 9 had other types of unresectable or recurrent neoplasms. Patients were 36 to 91 years of age (average 65.2 years); 12 were men and 40 were women.

Breast cancer patients younger than 75 also received systemic chemotherapy (EC/Tx) prior to and/or following the KORTUC II treatment.

The ethics committee of the Medical School of Kochi University approved KORTUC II for advanced skin cancer including malignant melanoma, bone/soft tissue malignant neoplasms, breast cancer, and metastatic lymph nodes.

Intratumoral injection of the radiosensitizer. The agent is composed of $0.5 \%$ hydrogen peroxide and $0.83 \%$ sodium hyaluronate, which is safe for injection, effectively preserving oxygen concentration in the tumor tissue for more than $24 \mathrm{~h}$ following intratumoral injection (10) while avoiding irritation of the local tissue. Injection of the agent was started in the second week of radiation therapy to avoid viable cancer cells migrating into micro-vessels. A maximum of $6 \mathrm{ml}$ was injected into the tumor tissue twice per week under ultrasonographic guidance, just prior to each administration of radiation therapy.

Radiation therapy. Radiation therapy with high-energy X-ray was delivered with an EXL-20TP linear accelerator (Mitsubishi Electric Co., Ltd., Tokyo, Japan) equipped with a multi-leaf collimator at an energy level appropriate for the depth of each lesion.

For patients with breast cancer, hypofraction radiotherapy was given using a tangential field approach; energy level was $4 \mathrm{MV}$, total radiation dose was $49.5 \mathrm{~Gy}$, administered as $2.75 \mathrm{~Gy} /$ fraction, five times per week. For those with cancers other than breast cancer, the total dose was $54 \mathrm{~Gy}$, with each fraction size being 2 Gy. For patients with cervical lymph node metastases or skin cancer, a total dose of $48 \mathrm{~Gy}$ of high energy electrons at an appropriate energy level was administered at $4 \mathrm{~Gy} /$ fraction, three times per week.

Assessment of therapeutic response. Tumor response was assessed according to the RECIST criteria (12), and patient monitoring and tumor assessment were carried out once a month. Patients were assigned a toxicity grade from a standard 
A

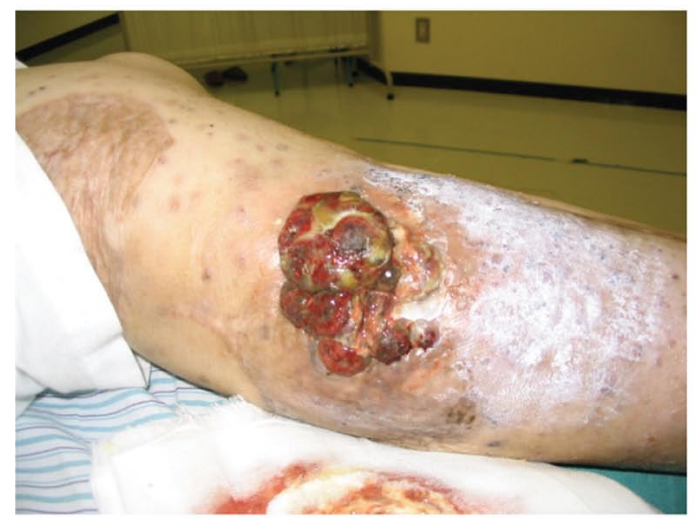

C

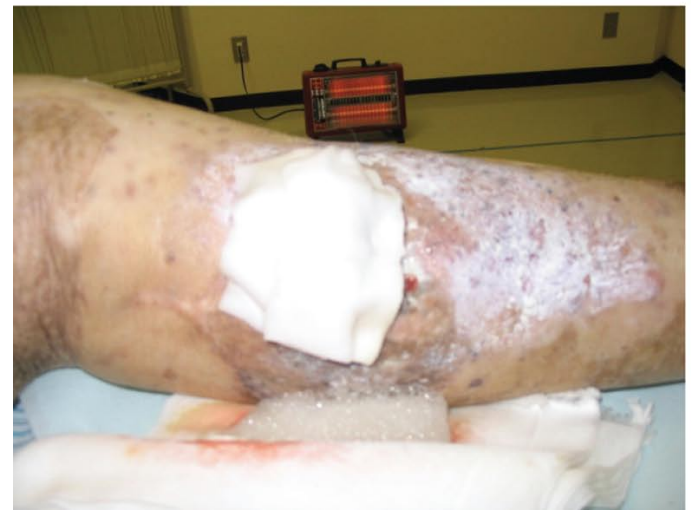

B

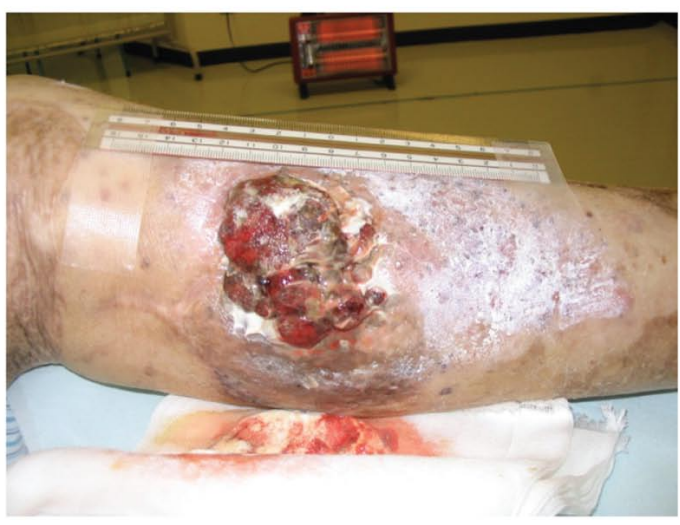

D

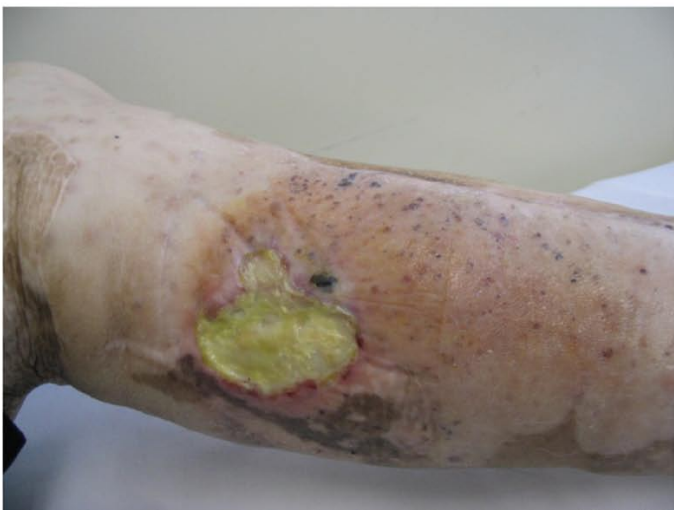

Figure 1. An example of KORTUC I treatment. For a case of recurrent and inoperable locally advanced malignant melanoma, sterilized cotton soaked in a hydrogen peroxide solution having a concentration of approximately $3 \% \mathrm{w} / \mathrm{v}$ was used as a bolus, and irradiation by an electron beam from a linear accelerator (KORTUC I radiosensitization therapy) was then conducted. The malignant melanoma was approximately $8 \mathrm{~cm}$ long before radiotherapy (A). Irradiation was then conducted three times a week for 4 weeks, with each radiation dose amounting to $4 \mathrm{~Gy}$ (12 times, for a total radiation dosage of $48 \mathrm{~Gy}$ ). In spite of 5 times of $4 \mathrm{~Gy}$ irradiation, the tumor showed no shrinkage (B). At that time, KORTUC I radiosensitization therapy was started. Specifically, sterilized cotton soaked in a hydrogen peroxide solution having a concentration of approximately $3 \% \mathrm{w} / \mathrm{v}$ was applied to the affected area of the locally advanced malignant melanoma as a bolus. After totally covering and gently massaging for several minutes with $3 \% \mathrm{w} / \mathrm{v}$ hydrogen peroxide solution (Oxydol)-soaked gauze, abundant bubbles of oxygen produced by the degradation of hydrogen peroxide by peroxidase were produced (C). The tumor had shrunk to approximately 5 cm at 2 weeks after termination of KORTUC I. At 3 months after termination of KORTUC I, the tumor had completely disappeared (D).

assessment scale (NIH common toxicity criteria). Treatmentrelated complications were assessed in detail to evaluate the feasibility of this approach. These patients were followed up for more than 12 months.

Formulation example. We used 1 syringe $(2.5 \mathrm{ml})$ of a hyaluronic acid preparation having a $1 \% \mathrm{w} / \mathrm{v}$ concentration of sodium hyaluronate (ARTZ Dispo, Seikagaku Corporation, Tokyo, Japan). This contained $25 \mathrm{mg}$ of sodium hyaluronate, $2.5 \mathrm{mg}$ of L-methionine, sodium chloride, potassium phosphate, crystalline sodium dihydrogen phosphate, and an isotonizing agent. The preparation is a colorless, transparent, viscous, aqueous solution having a $\mathrm{pH}$ of 6.8 to 7.8 , specific osmotic pressure of 1.0 to 1.2 (relative to physiological saline), and a weight-average molecular weight of 600,000 to 1.2 million. To this, $0.5 \mathrm{ml}$ of a $3 \% \mathrm{w} / \mathrm{v}$ solution of hydrogen peroxide (Oxydol, Ken-ei Pharmaceutical Co. Ltd., Osaka, Japan) was added immediately before use, and mixed well to prepare the radiosensitizer. The sensitizer has a sodium hyaluronate concentration of $0.83 \%$ and a hydrogen peroxide concentration of approximately $0.5 \%$. This preparation was used in the study.

\section{Results}

Patients treated with KORTUC II. After providing fully informed consent, 52 patients with unresectable or recurrent malignant neoplasms were enrolled in the KORTUC II trial.

Treatment was well tolerated, with a minimum of adverse effects. Of the 53 lesions in the 52 patients, 30 (57\%) showed complete response (CR), 14 (26\%) revealed partial response (PR), 6 (11\%) showed no change (NC), and another 3 (6\%) (bone metastases) were not evaluable. Overall survival rate was $73.9 \%$ at 1 year (34/46 patients) and $50.0 \%$ at 2 years (15/30 patients). Disease-free survival rate was $58.7 \%$ at 1 year (27/46 patients) and $36.7 \%$ at 2 years (11/30 patients).

Adverse events were experienced by 28 patients. All were minor, consisting of mild local pain at the injection site. For all 


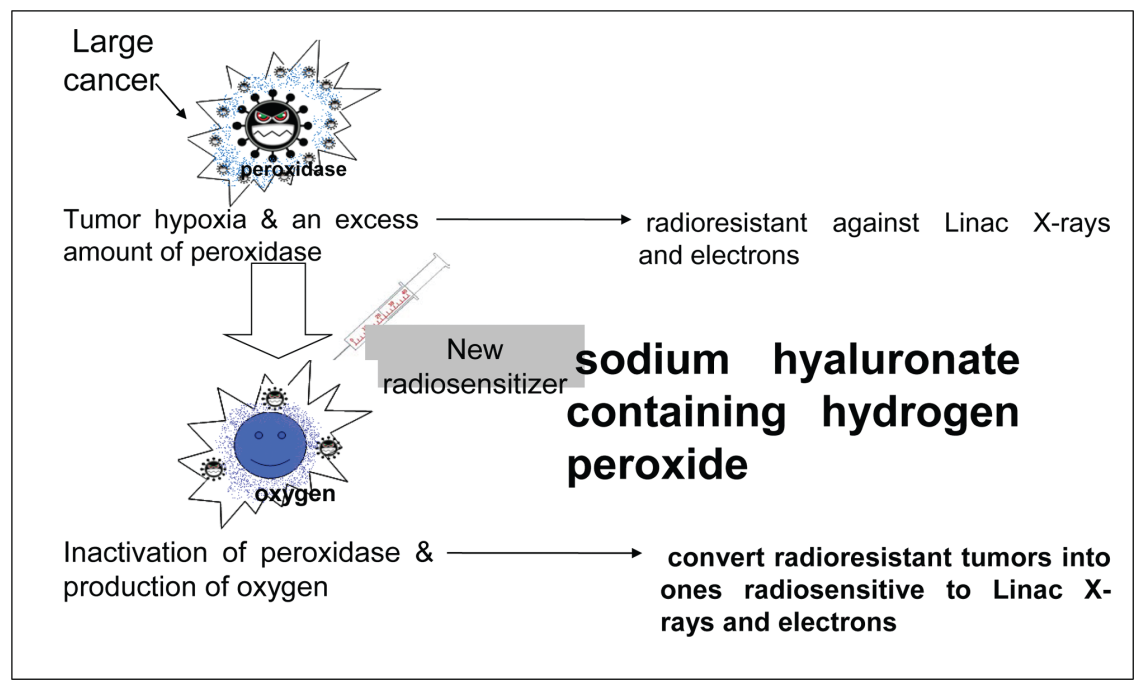

Figure 2. Concept of the new enzyme-targeting radiosensitization treatment (KORTUC II).

A

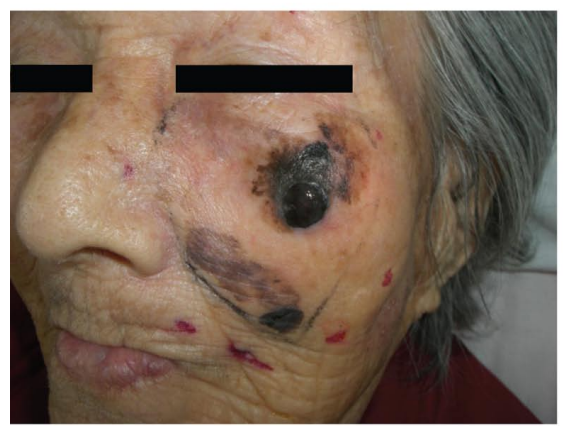

C

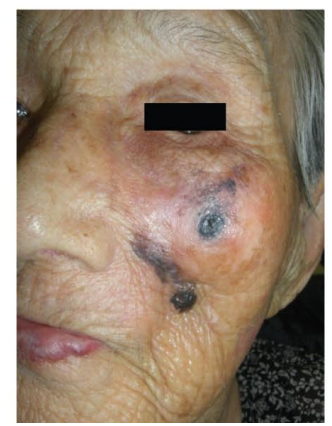

B

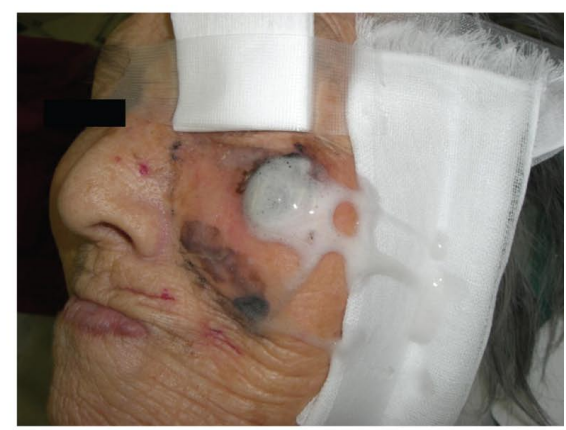

D

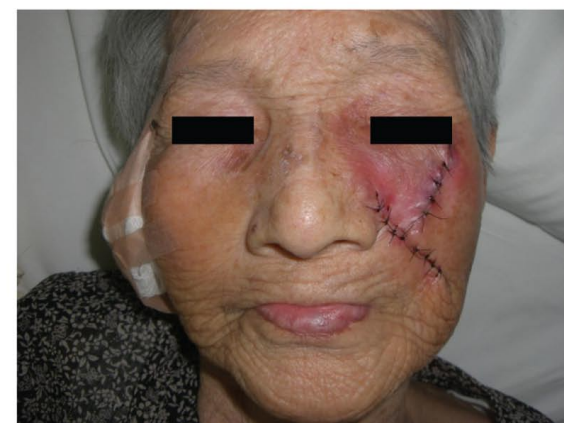

Figure 3. A 96-year-old woman with locally advanced malignant melanoma who underwent KORTUC II treatment. (A) Before treatment. (B) Oxygen production after injection of the agent. (C) Six months following KORTUC II. (D) pCR (pathologically complete response) was confirmed from the pathological specimen obtained from limited resection.

52 patients, radiation dermatitis was mild (Grade I) and equivalent to that observed at our facility after radiation therapy alone.

Examples of low-LET radioresistant tumors for which KORTUC II treatment produced remarkable therapeutic effects. Case 1. A 96-year-old woman with locally advanced malignant melanoma (Fig. 3). She underwent intratumoral injection of $3 \mathrm{ml}$ of the radiosensitizer, followed by irradiation. Oxygen production was seen following the injection of the radiosensitizer (B). The malignant melanoma was irradiated three times a week for 4 weeks, with each of the 12 doses being $4 \mathrm{~Gy}$, for a total dosage of $48 \mathrm{~Gy}$. Nine months after the KORTUC II treatment, limited resection was performed (D), and pathological examination demonstrated pCR (pathologically complete response).

Case 2. A 70-year-old man with right axillary metastasis of renal cell carcinoma treated with KORTUC II (Fig. 4). Before treatment, a huge metastatic lymph node of more than $10 \mathrm{~cm}$ in long diameter was evident in the right axillary region (A). Six months following KORTUC II treatment, the tumor 
A

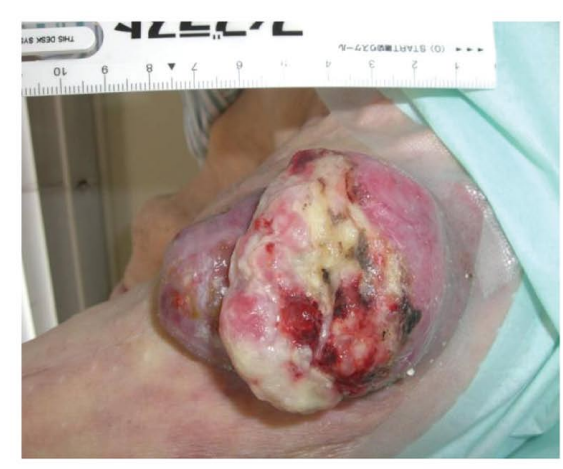

C

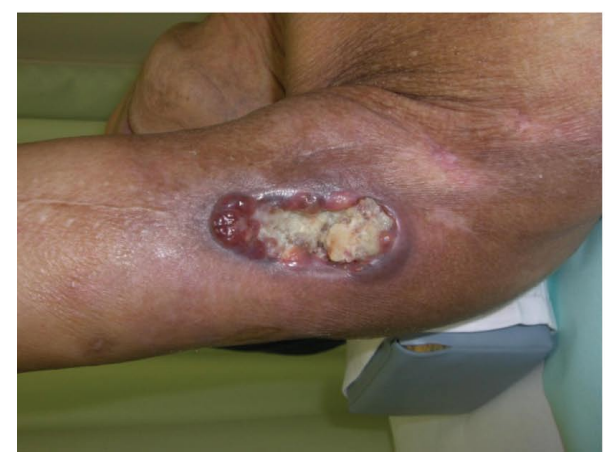

B

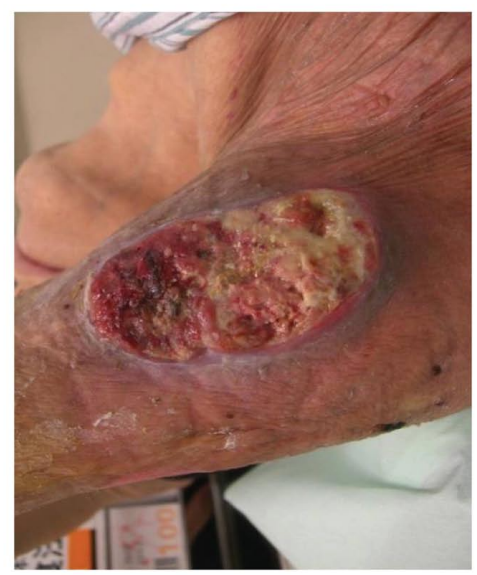

D

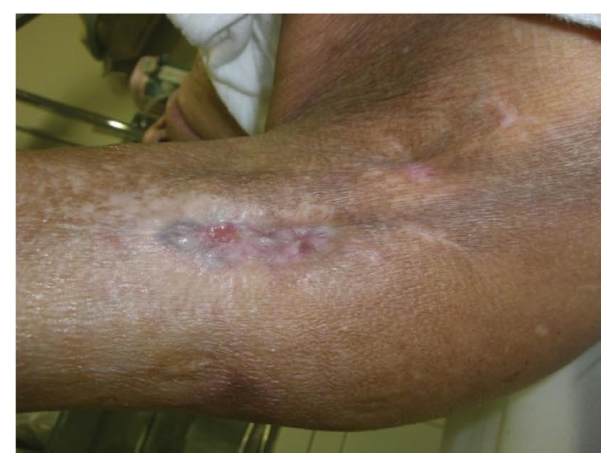

Figure 4. A 70-year-old man with right axillary metastasis of renal cell carcinoma treated with KORTUC II. (A) Before treatment; (B) after the course of irradiation (54 Gy). (C) Three months following KORTUC II (D) 6 months following KORTUC II, the tumor completely disappeared and was evaluated as cCR (clinically complete response).

completely disappeared and was evaluated as cCR (clinically complete response) (D).

Case 3. A 78-year-old man with recurrent fibrosarcoma of the right leg (Fig. 5). Magnetic resonance imaging (MRI) performed 3 months prior to treatment showed a tumor of approximately $2 \mathrm{~cm}$ in thickness (A). MRI just prior to KORTUC II treatment showed that the tumor had grown rapidly in the previous 3 months (B). MRI 6 months following KORTUC II treatment demonstrated cessation of tumor growth (C). MRI 11 months following KORTUC II treatment showed shrinkage of more than $50 \%$ of the tumor compared to the pre-treatment size, and the case was evaluated as cPR (clinically partial response) (D).

Case 4. A 74-year-old man with locally advanced facial skin cancer (SCC) (Fig. 6) before KORTUC II (A) and 1 month after KORTUC II (B). The huge tumor, which initially measured more than $10 \mathrm{~cm}$ in diameter, showed remarkable shrinkage of more than $90 \%$ compared to its pre-treatment size, and was evaluated as cPR.

\section{Discussion}

Currently, most radiation therapy is performed using X-rays or high-energy electron beams from a linear accelerator; however, these forms of radiation are not ideal in terms of their low-linear energy transfer (LET) characteristics. Almost two-thirds of the radiation-induced damage to cancer cells caused by these low-LET results from the production of reactive oxygen species (ROS) in irradiated cells (3). Therefore, oxygen is essential for fixation of the radicals produced by irradiation. In contrast, antioxidative enzymes such as peroxidase/catalase scavenge radicals produced by low-LET irradiation, resulting in a decreased irradiation effect. Peroxidase/catalase is abundant in most tumor tissues, as demonstrated by the fact that intrinsic peroxidase must be blocked before immunohistochemical staining of tumor tissue $(13,14)$.

Examples of tumors that are low-LET radioresistant include those that have many hypoxic tumor cells or abundant antioxidative enzymes. The low-LET radioresistant tumor cells are mostly in hypoxic regions of the tumor. In the hypoxic state, DNA damage induced by the radiation is not oxidized. Moreover, reactive oxygen species produced in the cancer cells by the radiation are eliminated by anti-oxidative enzymes, and this appears to impede apoptosis induced by dysfunction of mitochondrial membrane potential or lysosomal membrane permeability. Specific examples of tumors that are low-LET radioresistant include malignant melanomas, glioblastoma multiforme, and various types of sarcomas such as osteosarcoma, as well as nearly all locally advanced neoplasms that have grown to several centimeters or more. 
A

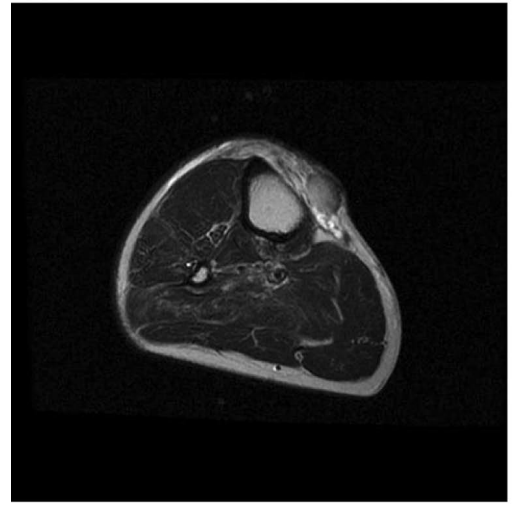

C

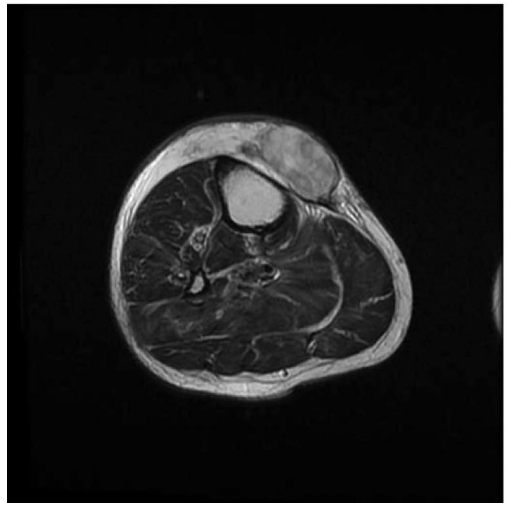

B

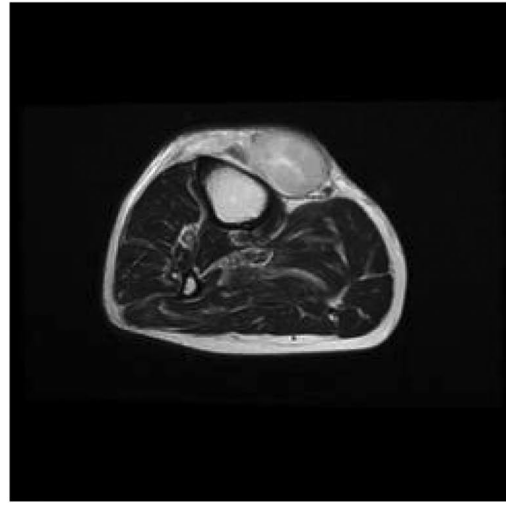

D

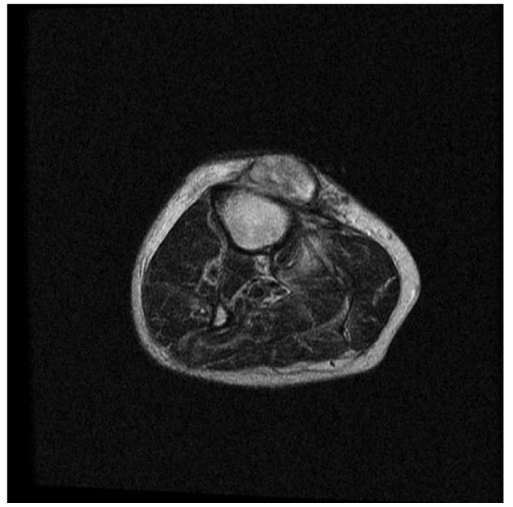

Figure 5. A 78-year-old man with recurrent fibrosarcoma of the right leg. (A) Magnetic resonance image (MRI) 3 months prior to treatment. (B) MRI just prior to KORTUC II treatment. The tumor showed rapid growth in the previous 3 months. (C) MRI 6 months following the KORTUC II treatment. Tumor growth had stopped. (D) MRI 11 months following KORTUC II treatment. The tumor showed shrinkage of more than 50\% compared to its pre-treatment size, and was evaluated as cPR (clinically partial response).

A

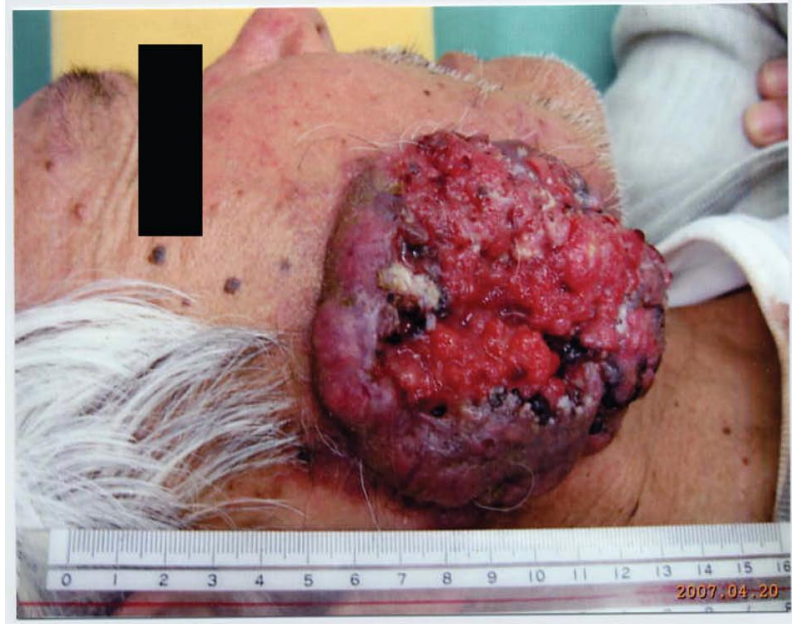

B

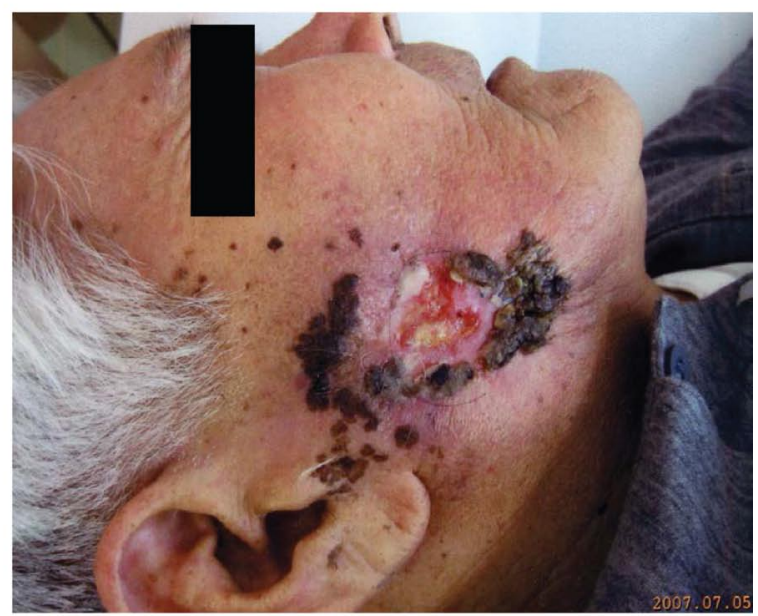

Figure 6. Photographs of a 74-year-old man with locally advanced facial skin cancer (SCC) before KORTUC II (A) and 1 month after KORTUC II (B). After KORTUC II treatment, the huge tumor measuring more than $10 \mathrm{~cm}$ in diameter showed shrinkage of more than $90 \%$ compared to the pre-treatment size, and was evaluated as cPR.

Although various radiosensitizing treatments for radioresistant neoplasms have been developed (15-19), a clinically applicable method has yet to be established. Many studies have examined the utility of increasing tissue oxygen concentrations to promote the fixation of radicals produced by low-LET irradiation to cancer cells/tissues, but few therapeutic gains have 
thus far been observed (20-22). Besides increasing tissue oxygen concentrations, it is considered important to inactivate antioxidative enzymes such as peroxidase and catalase. Hydrogen peroxide is the only agent that both inactivates anti-oxidative enzymes and produces oxygen when applied topically to tumor tissue.

When treating low-LET radioresistant tumors, it is therefore necessary to develop a new method that both inactivates antioxidative enzymes and produces oxygen in tumor tissue to augment the effects of X-rays and high-energy electron beams.

Regarding intratumoral hydrogen peroxide injection, 3-5 ml of approximately $3 \% \mathrm{w} / \mathrm{v}$ hydrogen peroxide solution (Oxydol) was administered for meningioma with no significant side effects (23). The present method, KORTUC II, uses only 3-6 ml of diluted Oxydol $(0.5 \% \mathrm{w} / \mathrm{v}$ hydrogen peroxide $)$ for intratumoral injection; thus, our dose of hydrogen peroxide is much lower than in the method described above (23).

Moreover, the combined use of sodium hyaluronate with hydrogen peroxide preserved oxygen concentration in tumor tissue for more than $24 \mathrm{~h}$, while intratumoral injection of hydrogen peroxide alone resulted in rapid lowering of oxygen concentration in the tumor tissue (unpublished data). Recently, injection of hydrogen peroxide into pelvic soft tissue between the rectum and urinary bladder was reported to have been safely performed for radiation therapy for prostate cancer, in terms of avoiding late side effects resulting from over-dose irradiation of the rectal wall (24).

The newly developed radiation sensitizer is a pharmaceutical compound that can be used on a tumor area prior to, or simultaneously with, radiotherapy to strengthen the effect that radiotherapy has on the tumor. Its application enables a superior radiotherapeutic effect using low-LET beams, even for low-LET radioresistant tumors, because they contain many hypoxic tumor cells and abundant peroxidase/catalase.

KORTUC II represents a novel form of treatment using a new radiosensitizer containing hydrogen peroxide and sodium hyaluronate that is injected intratumorally, mainly under ultrasonographic guidance. The treatment was well tolerated, with a minimum of adverse effects such as local pain at the injection site.

The method is targeted at intrinsic peroxidase and catalase present in tumor cells, infiltrating granulocytes, and contaminating red blood cells in the tumor tissue. Therefore, it is considered to be a new enzyme-targeting radiosensitization treatment potentially indicated for almost every type of low-LET radioresistant neoplasm.

Radiotherapy using this radiosensitizer can be implemented by first applying the radiosensitizer to the area of the tumor to be irradiated, and then preferably using a linear accelerator to irradiate the affected part with X-rays or electron beams. Regarding use of the agent, it is essential to avoid direct injection into blood vessels and to confirm even distribution of oxygen micro-bubbles throughout the tumor tissue using ultrasonographic or computer tomographic guidance. Hence, KORTUC II can be thought of as a new image-guided radiosensitization method.

Because this radiosensitizer is safe and less expensive than other methods, and can be applied for almost every type of low-LET radioresistant neoplasm, it has potential for worldwide and immediate use.

\section{Acknowledgments}

We thank Mr. N. Yokota and Mr. T. Sasaki for technical assistance. This study was supported by a grant-in-aid for Scientific Research from the Japanese Ministry of Education, Culture, Sports, Science and Technology (21591610). The sponsor had no role in study design, data collection, data analysis, data interpretation or writing of the manuscript. Y.O. and K.K. performed project planning; Y.O., K.K., H.U., M.T., R.M., T.Y., N.H., S.K., A.N., H.N., M.T., and S.S performed the treatment; Y.O. wrote the manuscript. The authors declare no competing financial interests.

\section{References}

1. Jette DC, Wiebe LI and Chapman JD: Synthesis and in vivo studies of the radiosensitizer 4-[82Br]bromomisonidazole. Int J Nucl Med Biol 10: 205-210, 1983.

2. Coleman CN: Hypoxic cell radiosensitizers: Expectations and progress in drug development. Int J Radiat Oncol Biol Phys 11: 323-329, 1985.

3. Hall EJ. The oxygen effect and reoxygenation. In: Radiobiology for the Radiologist. 5th edition. J.B. Lippincott Co., Philadelphia, PA, pp91-111, 2000.

4. Ogawa Y, Takahashi T, Kobayashi T, et al: Mechanism of apoptotic resistance of human osteosarcoma cell line, HS-Os-1, against irradiation. Int J Mol Med 12: 453-458, 2003.

5. Ogawa Y, Takahashi T, Kobayashi T, et al: Apoptotic-resistance of the human osteosarcoma cell line HS-Os-1 to irradiation is converted to apoptotic-susceptibility by hydrogen peroxide: a potent role of hydrogen peroxide as a new radiosensitizer. Int J Mol Med 12: 845-850, 2003.

6. Ogawa Y, Takahashi T, Kobayashi T, et al: Immunocytochemical characteristics of human osteosarcoma cell line HS-Os-1: Possible implication in apoptotic resistance against irradiation. Int J Mol Med 14: 397-403, 2004.

7. Kariya S, Sawada K, Kobayashi T, et al: Combination treatment of hydrogen peroxide and $\mathrm{X}$-rays induces apoptosis in human prostate cancer PC-3 cells. Int J Radiat Oncol Biol Phys 75: 449-454, 2009.

8. Ogawa Y, Takahashi T, Kobayashi T, et al: Comparison of radiation-induced reactive oxygen species formation in adult articular chondrocytes and that in human peripheral T cells: Possible implication in radiosensitivity. Int J Mol Med 11: 455-459, 2003.

9. Ogawa Y, Ue H, Tsuzuki K, et al: New radiosensitization treatment (KORTUC I) using hydrogen peroxide solution-soaked gauze bolus for unresectable and superficially exposed neoplasms. Oncol Rep 19: 1389-1394, 2008.

10. Ogawa Y, Kubota K, Ue H, et al: Development and clinical application of a new radiosensitizer containing hydrogen peroxide and hyaluronic acid sodium for topical tumor injection - a new enzyme-targeting radiosensitization treatment, KORTUC II (Kochi Oxydol-Radiation Therapy for Unresectable Carcinomas, Type II). Strahlenther. Onkol 183 (Sondernr. 2): 100-101, 2007.

11. Ogawa Y, Kubota K, Ue H, et al: Phase I study of a new radiosensitizer containing hydrogen peroxide and sodium hyaluronate for topical tumor injection: A new enzyme-targeting radiosensitization treatment, Kochi Oxydol-Radiation Therapy for Unresectable Carcinomas, Type II (KORTUC II). Int J Oncol 34: 609-618, 2009.

12. Therasse P, Arbuck SG, Eisenhauer EA, et al: New guidelines to evaluate the response to treatment in solid tumors. J Natl Cancer Inst 92: 205-216, 2000.

13. Ogawa Y, Nishioka A, Hamada N, et al: Immunohistochemical study of c-fos-positive lymphocytes infiltrated into human squamous cell carcinomas of the head and neck during radiation therapy and its clinical significance. Clin Cancer Res 3: 2301-2307, 1997.

14. Ogawa Y, Nishioka A, Hamada N, et al: Expression of Fas (CD95/APO-1) antigen induced by radiation therapy for diffuse B-cell lymphoma: immunohistochemical study. Clin Cancer Res 3: 2211-2216, 1997.

15. Lawrence TS, Blackstock AW and McGinn C: The mechanism of action of radiosensitization of conventional chemotherapeutic agents. Semin Radiat Oncol 13: 13-21, 2003. 
16. Bohm L, Roos WP and Serafin AM: Inhibition of DNA repair by pentoxifylline and related methylxanthine derivatives. Toxicol 193: 153-160, 2003.

17. Milas L: Cyclooxygenase-2 (COX-2) enzyme inhibitors as potential enhancers of tumor radioresponse. Semin Radiat Oncol 11: 290-299, 2001.

18. Choy H and Milas L: Enhancing radiotherapy with cyclooxygenase-2 enzyme inhibitors: a rational advance? J Natl Cancer Inst 95: 1440-1452, 2003.

19. Overgaad J: Clinical evaluation of nitroimidazoles as modifiers of hypoxia in solid tumors. Oncol Res 6: 509-518, 1994.

20. Gerlach NL, Barkhuysen R, Kaanders JH, et al: The effect of hyperbaric oxygen therapy on quality of life in oral and oropharyngeal cancer patients treated with radiotherapy. Int J Oral Maxillofac Surg 37: 255-259, 2008.
21. Overgaad J: Hypoxic radiosensitization: adored and ignored. J Clin Oncol 25: 4066-4074, 2007.

22. Williamson RA: An experimental study of the use of hyperbaric oxygen to reduce the side effects of radiation treatment for malignant disease. Int J Oral Maxillofac Surg 36: 533-540, 2007.

23. Lichtenbaum R, De Souza AA and Jafar JJ: Intratumoral hydrogen peroxide injection during meningioma resection. Neurosurgery 59 (ONS Suppl. 4): S470-S473, 2006.

24. Prada PJ, Fernandez J, Martinez AA, et al: Transperineal injection of hyaluronic acid in anterior perirectal fat to decrease rectal toxicity from radiation delivered with intensity-modulated brachytherapy or EBRT for prostate cancer patients. Int J Radiat Oncol Biol Phys 69: 95-102, 2007. 